\title{
Comparison of Two Cyberknife Planning Approaches for Multiple Brain Metastases
}

\author{
Tianlong Ji, Yaowen Song, Xinyu Zhao, Yuzi Wang and Guang Li* \\ Department of Radiation Oncology, The First Hospital of China Medical University, Shenyang, China
}

OPEN ACCESS

Edited by:

Yidong Yang,

University of Science and Technology

of China, China

Reviewed by:

John E. Mignano,

Tufts University School of Medicine,

United States

Yibao Zhang,

Peking University Cancer Hospital,

China

${ }^{*}$ Correspondence:

Guang Li

dr_liguang@163.com

Specialty section:

This article was submitted to

Radiation Oncology,

a section of the journal

Frontiers in Oncology

Received: 18 October 2021

Accepted: 10 January 2022

Published: 03 February 2022

Citation:

Ji T, Song $Y$, Zhao $X$, Wang $Y$ and $L i G$ (2022) Comparison of Two

Cyberknife Planning Approaches for Multiple Brain Metastases.

Front. Oncol. 12:797250.

doi: 10.3389/fonc.2022.797250
Purpose: To compare the delivery efficiency, plan quality, and planned treatment volume (PTV) and normal brain dosimetry between different Cyberknife planning approaches for multiple brain metastases (MBM), and to evaluate the effects of the number of collimators on the related parameters.

Methods: The study included 18 cases of MBM. The Cyberknife treatment plans were classified as Separate or Combined. For the Separate plan, each lesion was targeted by the collimator auto-selection method (Conformality 2/3 collimators). For the Combined plan, a PTV including all PTVs was targeted by the collimators. Monitor units (MUs), number of nodes and beams, estimated fraction treatment time (EFIT), new conformity index $(\mathrm{nCl})$, dose gradient index $(\mathrm{Gl})$, homogeneity index $(\mathrm{HI})$, PTV minimum/maximum dose $\left(D_{\max } / D_{\min }\right)$, volume doses $\left(D_{2 \%}\right.$ and $\left.D_{98 \%}\right)$, maximum doses to lenses, optic nerves, and brainstem as well as normal brain 3, 6, 10, and 12 Gy ( $\left.V_{3 G y}-V_{12 G y}\right)$ were compared.

Results: Compared to the Combined plan, the Separate plan had fewer nodes and beams, shorter EFIT, smaller PTV D min, normal brain dose, and Gl, and larger HI. The Separate plan with 2 collimators also had worse PTV coverage. In the Combined plan, more collimators increased beams, EFT, Gl, and normal brain dose but improved the PTV $D_{\min }$. Among treatments based on the Separate approach, there were obvious differences between plans for most of the items except the $\mathrm{nCl}$. Fewer collimators resulted in significantly reduced beams, EFT, PTV D98\%, and normal brain dose with improved Gl, although PTV $D_{\min }$ and MUs were decreased while HI was increased.

Conclusion: Both approaches met the requirements for SRS/HFSRT. We found that Separate plans improved treatment efficiency and normal tissue dosimetry.

Keywords: Cyberknife, Iris collimator, treatment planning, stereotactic radiosurgery, multiple brain metastases 


\section{INTRODUCTION}

Stereotactic radiosurgery (SRS) and hypo-fractionated stereotactic radiotherapy (HFSRT) are efficient and welltolerated treatment modalities for patients with brain metastases $(1,2)$. In patients with multiple brain metastases (MBM), both techniques have proven effective for reducing neurotoxicity and preserving quality of life (3-5). SRS and HFSRT can be performed with a Gamma Knife (GK; Elekta $\mathrm{AB}$; Stockholm, Sweden), a Cyberknife robotic radiosurgery system (CK; Accuray, Sunnyvale, CA, USA), or a conventional linear accelerator, and in the treatment of MBM, there may be marked differences in plan quality and delivery efficiency among these devices $(6,7)$.

The delivery and plan optimization features of the CK have been associated with excellent overall survival and local control rates in MBM, with relatively low toxicity (8). The CK has a 6$\mathrm{MV}$ accelerator mounted on a robotic arm that is designed to deliver non-isocenter non-coplanar beam arrangements; this is combined with a high-resolution image-guided tracking system to help maximize the accuracy of the treatment. The positions of the nodes are fixed, and the beams vary according to the target.

Regardless of the equipment, different treatment planning approaches will lead to differences in plan quality and delivery efficiency $(9,10)$. The CK Model $\mathrm{M}^{\mathrm{TM}}{ }^{\mathrm{T}}$ that was evaluated in this research is equipped with a fixed cones collimator as well as an Iris $^{\mathrm{TM}}$ variable aperture collimator. The variable aperture collimator can provide better delivery efficiency than the fixed cone collimator by freely changing collimator sizes. Generally, the collimator selection is based on experience. It can also depend on the recommendations generated by a treatment planning system (TPS; e.g., Multiplan v5.3). Figure 1 shows a TPS-generated collimator selection. The selection of collimators can affect the target coverage, the sparing of organs at risk (OARs), and the delivery efficiency (11).
Treatment planning for MBM is particularly complex because of the close proximity of the treatment targets to neighboring critical and radiosensitive structures (12). The number of contours, including the OARs and the target, is limited to 23 in the TPS, and the extension of each lesion to a separate planned treatment volume (PTV) is limited, especially in cases of more than 5 lesions. Because of this, a PTV including all lesions is sometimes used for the process of collimator selection, rather than basing the collimator selection on each lesion separately.

The purpose of this study was to compare CK treatment planning approaches for MBM in terms of the delivery efficiency, dosimetry of PTV and normal brain, and plan quality. In addition, for each approach, we compared two collimator selection methods with different numbers and sizes of Iris collimators in order to evaluate the effects of the number of collimators on the dosimetry.

\section{METHODS}

\section{Lesions}

Eighteen patients treated for MBM with the CK Model $\mathrm{M}^{\mathrm{TM}}$ were included in this study. Lesion numbers varied from two to six per patient, and a total of 73 lesions were enrolled (Table 1).

TABLE 1 | Detailed tumor information.

\begin{tabular}{llc} 
Item & & Value \\
\hline Number of patients & & 18 \\
Number of lesions: & Total & 73 \\
& Median & 4 \\
Volume of lesions $\left(\mathrm{cm}^{3}\right):$ & Min & 0.409 \\
& Max & 26.645 \\
& Average for lesions & 5.837 \\
& Average for cases & 23.828
\end{tabular}

B

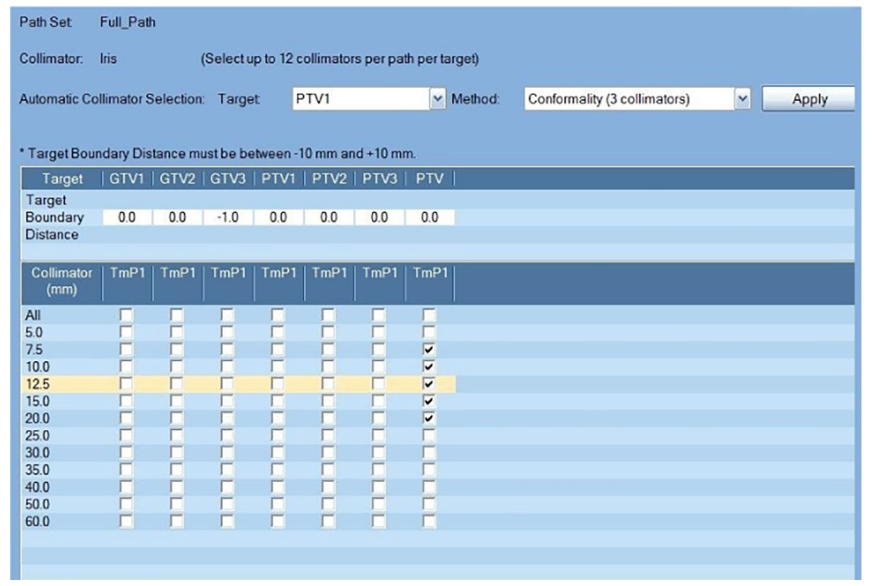

FIGURE 1 | Comparison of two different treatment planning approaches. Lesions were targeted separately (A) or all of the lesions were included in a single PTV (B); the "Conformality(3collimator)" auto-selection method was applied for the two planning approaches in this example. 
Contour delineation was based on 1-mm CT and MRI slice thickness. The PTV was defined as the gross tumor volume (GTV) plus $1 \mathrm{~mm}$ for all plans. Each GTV was extended separately to generate a PTV, and a PTV all including all PTVs was then generated for this analysis. The OARs included skin, normal brain, eyes, lenses, brainstem, cochlea, and optic nerves. The normal brain area was defined as the whole brain minus $\mathrm{PTV}_{\text {all }}$. Detailed tumor information is presented in Table $\mathbf{1 .}$

\section{Treatment Plans}

Four plans were made for each case according to either the Separate or the Combined approach, as described below. The prescription dose was $35 \mathrm{~Gy} / 5 \mathrm{~F}$ for all cases. The beam intersection model was set to exclude the eyes and the brainstem. The optimization goal for the PTV was set as optimized minimum dose (OMI) equal to the prescription dose. All treatment plans implemented time reduction $(70 \%$ the time evaluated at first optimization) and were normalized at 95\% PTV covered by $100 \%$ prescription dose.

For the Separate approach (Sep), PTVs were selected separately as the target in the model of collimator selection (Figure 1A). For the Combined approach (All), PTV $\mathrm{All}_{\text {ll }}$ was selected as the target in the model of collimator selection. To pick the correct collimator size, conformality(2or3 collimator) auto-selection methods with 2 and 3 collimators were applied for each approach (Figure 1). Accordingly, the corresponding treatment plans for each approach are named Sep_2 and Sep_3 and All_2 and All_3.

\section{Plan Evaluation}

The plans were evaluated for monitor units (MUs); number of nodes and beams; estimated fraction treatment time (EFTT); new conformity index ( $\mathrm{nCI}$ ); dose gradient index (GI); homogeneity index (HI); dose-volume histogram (DVH) parameters for PTV including minimum dose $\left(\mathrm{D}_{\min }\right)$ and maximum dose $\left(\mathrm{D}_{\max }\right)$; the $2 \%$ PTV maximum dose $\left(\mathrm{D}_{2 \%}\right)$; and the $98 \%$ PTV minimum dose $\left(\mathrm{D}_{98 \%}\right)$. For normal brain tissue, parameters including volumes receiving a specific dose at 3, 6, 10, and $12 \mathrm{~Gy}\left(\mathrm{~V}_{3 \mathrm{~Gy}}, \mathrm{~V}_{6 \mathrm{G} y}, \mathrm{~V}_{10 \mathrm{~Gy}}, \mathrm{~V}_{12 \mathrm{~Gy}}\right)$ were evaluated. Maximum doses to lenses, optic nerves, and brainstem were also evaluated. The optic chiasm is included with the same dose limitation setting in our department.

The EFTT was evaluated by the TPS according to the number of nodes, beams, and MUs along with a user-defined estimated patient setup time and estimated image time interval.
The nCI was used to evaluate the conformity of the dose distribution and calculated as: $\mathrm{nCI}=\left(\mathrm{V}_{\mathrm{t}}{ }^{*} \mathrm{~V}_{\mathrm{p}}\right) /\left(\mathrm{V}_{\mathrm{tp}}\right)^{2}$, where $\mathrm{V}_{\mathrm{tp}}$ is the PTV within the prescription isodose surface, $V_{t}$ is the PTV, and $V_{p}$ is the prescription volume (13).

$\mathrm{HI}$ indicates the degree of uniformity of dose distribution within the target and was calculated as: $\mathrm{HI}=$ (max dose/prescribed dose).

GI describes the steepness of the dose gradient and was calculated as: $\mathrm{GI}=\mathrm{V}_{50 \%} \mathrm{D}_{\mathrm{p}} / \mathrm{V}_{\mathrm{p}}$, where $\mathrm{V}_{50 \%} \mathrm{D}_{\mathrm{p}}$ is $50 \%$ of the prescription isodose line volume and $\mathrm{V}_{\mathrm{p}}$ is the prescription volume.

\section{Statistical Analysis}

All statistical analyses were performed with SPSS 20.0 (SPSS Inc., Chicago, IL, USA). All data were normalized by parametric tests to accommodate comparison by paired samples t-test. The Wilcoxon signed-rank test was considered the non-parametric counterpart of the paired t-test. $p<0.05$ was considered as statistically significant.

\section{RESULTS}

The treatment delivery efficiency parameters are shown in Table 2. Compared to the Combined plans, the Separate plans had fewer nodes (142.4 vs. 151.6 and 153.2 vs. $156.0 ; p=0.011$ and $<0.001)$ and beams (379.6 vs. 437.9 and 464.3 vs. 510.1 , $p<0.001)$ and shorter EFTTs (58.8 min vs. $63.7 \mathrm{~min}$ and 65.3 $\min$ vs. $68.9 \mathrm{~min}, p<0.001$ and 0.005$)$. There were no significant differences for MUs.

For the same approach with different collimator settings, smaller collimator values resulted in increased MUs $(54,497.6$ vs. $53,835.6$ and $55,910.5$ vs. $54,446.7, p=0.022$ and 0.016 ) with fewer beams (437.9 vs. 510.1 and 379.6 vs. $464.3, p<0.001$ ) and shorter EFTTs (63.7 min vs. $68.9 \mathrm{~min}$ and $58.8 \mathrm{~min}$ vs. $65.3 \mathrm{~min}$, $p<0.001)$ and fewer nodes (151.6 vs. 156.0 and 142.4 vs. 153.2, $p=0.011$ and 0.001$)$ in the Separate approach. There were no significant differences for the other metrics.

The Separate approach produced obviously lower PTV $\mathrm{D}_{\min }$ values (3132.1 cGy vs. 3015.6 cGy and 3197.8 cGy vs. 3149.5 cGy, $p=0.010$ and 0.026$)$ compared to the Combined approach. For treatment plans with 2 collimators, the Combined approach produced lower PTV $D_{\max }$ (4919.5 cGy vs. 5101.1 cGy, $p=$ 0.020 ) and $\mathrm{D}_{2 \%}$ (4581.1 cGy vs. 4788.1 cGy, $p=0.003$ ) values, respectively.

TABLE 2 | Treatment delivery efficiency: MUs, nodes, beams, and EFTT (minutes) among the four plans.

\begin{tabular}{|c|c|c|c|c|c|c|c|c|}
\hline & ALL_2 & ALL_3 & Sep_2 & Sep_3 & $\begin{array}{c}\text { ALL_2 VS } \\
\text { ALL_3 }\end{array}$ & $\begin{array}{l}\text { Sep_2 vS } \\
\text { Sept_3 }\end{array}$ & $\begin{array}{c}\text { ALL_2 VS } \\
\text { Sep_2 }\end{array}$ & $\begin{array}{c}\text { ALL_3 VS } \\
\text { Sep_3 }\end{array}$ \\
\hline MUs & $\begin{array}{c}54,497.6 \pm \\
6410.9\end{array}$ & $\begin{array}{c}53,835.6 \pm \\
6788.4\end{array}$ & $\begin{array}{c}55,910.5 \pm \\
7985.7\end{array}$ & $\begin{array}{c}54,446.7 \pm \\
6886.0\end{array}$ & $0.022^{a}$ & $0.016^{b}$ & $0.064^{b}$ & $0.107^{b}$ \\
\hline Nodes & $151.6 \pm 17.3$ & $156.0 \pm 11.6$ & $142.4 \pm 19.1$ & $153.2 \pm 12.6$ & $0.011^{b}$ & $<0.001^{a}$ & $0.001^{a}$ & $0.019^{a}$ \\
\hline Beams & $437.9 \pm 142.7$ & $510.1 \pm 168.4$ & $379.6 \pm 127.8$ & $464.3 \pm 145.8$ & $<0.001^{a}$ & $<0.001^{a}$ & $<0.001^{b}$ & $0.005^{b}$ \\
\hline $\mathrm{EFTT}$ (min) & $63.7 \pm 18.6$ & $68.9 \pm 20.6$ & $58.8 \pm 17.9$ & $65.3 \pm 19.0$ & $<0.001^{a}$ & $<0.001^{a}$ & $<0.001^{b}$ & $0.005^{a}$ \\
\hline
\end{tabular}

${ }^{a}$ Comparison by paired samples t-test.

${ }^{b}$ Comparison by Wilcoxon signed-rank test. 
The plans with two collimators using the Separate approach generated greater PTV $D_{\max }$ (5101.1 cGy vs. 4860.9 cGy, $p=$ 0.020 ) and $\mathrm{D}_{2 \%}$ (4788.1 cGy vs. 4528.5 cGy, $p=0.001$ ) values. Meanwhile, fewer collimators resulted in lower PTV $D_{\min }$ values (3132.1 cGy vs. 3197.8 cGy and 3015.6 cGy vs. 3149.5 cGy, $p=$ 0.005 and 0.002 ) for plans on both approaches. There were no significant differences for the other metrics. The details are shown in Table 3.

There were very clear differences among the plans for the protection of normal brain, and for the same number of collimators, the Separate approach produced lower normal brain doses. By the same approach, the fewer the collimators, the lower the dosimetry for normal brain.

These results are shown in Table 4.

As shown in Table 4, for the same number of collimators, the plans on the Separate approach showed obviously increased HI (1.46 vs. 1.41 and 1.37 vs. $1.36, p=0.016$ and 0.016 ) and decreased GI (4.10 vs. 4.49 and 4.75 vs. $5.06, p=0.014$ and 0.001) in comparison with the Combined approach. There were no significant differences in the nCI.

The plans with two collimators showed increased HI (1.41 vs. 1.36 and 1.46 vs. $1.37 p=0.016$ and 0.001$)$. Meanwhile, fewer collimators resulted in better GI (4.49 vs. 5.06 and 4.10 vs. 4.75 , $p<0.001)$ for plans on both approaches. There were no significant differences for the other metrics. The details are shown in Table 5.

\section{DISCUSSION}

Depending on lesion numbers and proximity of lesions to vital and radiosensitive structures, separate or combined PTVs can be defined for CK treatment planning in MBM. This study compared separate and combined planning approaches with the same optimization parameters for quality, delivery efficiency, and PTV and normal brain dosimetry and examined the impact of the number of collimators on the plans with different approaches.

There were significant differences between the planning approaches. With the same collimator selection, the separate approaches resulted in fewer beams and nodes, shorter EFTTs, decreased doses to normal brain, and lower GI than the combined approaches, although the numbers were a little worse for PTV $\mathrm{D}_{\min }$ and HI. A separate approach with two collimators also increased the PTV $\mathrm{D}_{\max }$ and $\mathrm{D}_{2 \%}$ and decreased the PTV $\mathrm{D}_{98 \%}$. This might be explained by the characteristics of the optimization algorithm. Schlaefer et al. (14) presented a stepwise optimization algorithm to optimize multiple clinical goals in steps with built-in priority.

TABLE 3 | DVH parameters for PTV among the four plans.

\begin{tabular}{|c|c|c|c|c|c|c|c|c|}
\hline & ALL_2 & ALL_3 & Sep_2 & Sep_3 & ALL_2 VS ALL_3 & Sep_2 VS Sept_3 & ALL_2 VS Sep_2 & ALL_3 VS Sep_3 \\
\hline Dmin (cGy) & $3132.1 \pm 105.1$ & $3197.8 \pm 80.3$ & $3015.6 \pm 152.7$ & $3149.5 \pm 91.0$ & $0.005^{a}$ & $0.002^{a}$ & $0.010^{a}$ & $0.026^{a}$ \\
\hline Dmax (cGy) & $4919.5 \pm 296.4$ & $4741.2 \pm 238.6$ & $5101.1 \pm 403.9$ & $4860.9 \pm 276.0$ & $0.172^{a}$ & $0.001^{b}$ & $0.020^{b}$ & $0.208^{a}$ \\
\hline $\mathrm{D}_{2 \%}$ (cGy) & $4581.1 \pm 40.2$ & $4472.4 \pm 236.1$ & $4788.1 \pm 330.6$ & $4528.5 \pm 275.0$ & $0.084^{a}$ & $0.001^{a}$ & $0.003^{b}$ & $0.106^{a}$ \\
\hline D98\% (cGy) & $3465.9 \pm 32.0$ & $3472.5 \pm 24.2$ & $3442.0 \pm 28.9$ & $3463.7 \pm 25.6$ & $0.378^{a}$ & $0.017^{a}$ & $0.015^{a}$ & $0.263^{a}$ \\
\hline
\end{tabular}

${ }^{a}$ Comparison by paired samples t-test.

${ }^{b}$ Comparison by Wilcoxon signed-rank test.

TABLE 4 | Dosimetric results for normal brain tissue, lens, optical nerves, and brainstem.

\begin{tabular}{|c|c|c|c|c|c|c|c|c|}
\hline & ALL_2 & ALL_3 & Sep_2 & Sep_3 & ALL_2 VS ALL_3 & Sep_2 VS Sept_3 & ALL_2 VS Sep_2 & ALL_3 VS Sep_3 \\
\hline V12Gy & $169.5 \pm 88.2$ & $192.4 \pm 98.6$ & $151.7 \pm 78.0$ & $172.4 \pm 80.0$ & $<0.001^{b}$ & $<0.001^{b}$ & $<0.001^{b}$ & $<0.001^{b}$ \\
\hline V10Gy & $220.3 \pm 119.3$ & $252.8 \pm 134.7$ & $193.6 \pm 100.0$ & $222.5 \pm 103.8$ & $<0.001^{b}$ & $<0.001 a$ & $<0.001^{b}$ & $0.001^{b}$ \\
\hline V6Gy & $439.4 \pm 213.6$ & $517.3 \pm 237.0$ & $386.8 \pm 180.0$ & $450.4 \pm 182.0$ & $<0.001^{b}$ & $<0.001 a$ & $0.002^{b}$ & $0.001^{b}$ \\
\hline V3Gy & $826.7 \pm 257.2$ & $925.4 \pm 253.9$ & $733.8 \pm 243.2$ & $861.2 \pm 238.4$ & $<0.001^{a}$ & $<0.001^{a}$ & $<0.001^{b}$ & $0.001^{a}$ \\
\hline Lens & $49.3 \pm 44.8$ & $56.2 \pm 21.9$ & $48.7 \pm 38.3$ & $62.4 \pm 34.5$ & $0.237^{b}$ & $0.398^{b}$ & $0.871^{b}$ & $0.310^{b}$ \\
\hline Nerves & $268.9 \pm 205.9$ & $370.9 \pm 282.1$ & $303.9 \pm 230.4$ & $337.3 \pm 244.9$ & $0.091^{b}$ & $0.499^{b}$ & $0.866^{b}$ & $0.735^{b}$ \\
\hline Brainstem & $546.5 \pm 502.6$ & $615.9 \pm 466.7$ & $515.4 \pm 380.2$ & $560.5 \pm 447.3$ & $0.028^{b}$ & $0.499^{b}$ & $0.612^{b}$ & $0.091^{b}$ \\
\hline
\end{tabular}

${ }^{a}$ Comparison by paired samples t-test.

${ }^{b}$ Comparison by Wilcoxon signed-rank test.

TABLE 5 | Dosimetric results for plan quality.

\begin{tabular}{|c|c|c|c|c|c|c|c|c|}
\hline & ALL_2 & ALL_3 & Sep_2 & Sep_3 & ALL_2 VS ALL_3 & Sep_2 VS Sept_3 & ALL_2 VS Sep_2 & ALL_3 VS Sep_3 \\
\hline $\mathrm{nCl}$ & $1.19 \pm 0.08$ & $1.19 \pm 0.07$ & $1.22 \pm 0.08$ & $1.15 \pm 0.08$ & $0.143^{a}$ & $0.072^{a}$ & $0.406^{b}$ & $0.263^{a}$ \\
\hline $\mathrm{HI}$ & $1.41 \pm 0.08$ & $1.36 \pm 0.07$ & $1.46 \pm 0.12$ & $1.37 \pm 0.08$ & $0.016^{a}$ & $0.001^{b}$ & $0.016^{b}$ & $0.016^{a}$ \\
\hline Gl & $4.49 \pm 0.77$ & $5.06 \pm 0.95$ & $4.10 \pm 0.60$ & $4.75 \pm 0.85$ & $<0.001^{b}$ & $<0.001^{a}$ & $0.014^{a}$ & $<0.001^{b}$ \\
\hline
\end{tabular}

${ }^{a}$ Comparison by paired samples t-test.

${ }^{b}$ Comparison by Wilcoxon signed-rank test. 
This was carried out in the CK planning system as the sequential optimizer. However, this algorithm cannot identify the spatial relationship between PTVs because all PTVs are projected onto one image in the process of optimization. The TPS optimizes the PTVs as a target that is irregular in shape and spatial position. Therefore, plans that combine PTVs may generate more and larger Iris collimators. Planning with combined PTVs resulted in more minimum and maximum sizes of the Iris collimators and the same appearance of MUs (Figure 2). A few studies have investigated the relationship of collimator diameter and the target coverage and have found that a larger collimator can reduce dose uncertainty and improve target coverage $(15,16)$. In this study, we found that planning for separate PTVs resulted in a slightly decreased minimum PTV dose. On the other hand, more beams through two PTVs introduced a higher dose to the space between the PTVs, which is why the normal brain dose is so high for the combined plans.

We also evaluated the effect of the number of collimators on the plan quality, delivery efficiency, and dosimetry with different optimization approaches. There was a significant difference in the number of nodes between the plans. Actually, the number of collimators per target may exceed 2 or 3 for the combined PTVs. Therefore, the two approaches demonstrated different effects. For the combined PTV approach, there were no significant differences in PTV $\mathrm{D}_{\max }, \mathrm{D}_{2 \%}, \mathrm{D}_{98 \%}$, and $\mathrm{nCI}$, which indicates that too many collimators might actually hinder improvement of the plan quality. More collimators also obviously increased nodes, beams, EFTT, GI, and dose to normal brain and decreased the MUs and HI, although improved PTV coverage produced slightly higher PTV $\mathrm{D}_{\text {min }}$. These results are consistent with those of Varnava et al., who conjectured that 2 collimators can do the same as the 10 collimators (17).

When planning for the separate PTVs, there were obvious differences between the plans with different numbers of collimators for most of the items, except the nCI. The plans with two collimators allowed significant reduction in the dose to normal tissue, with corresponding GI improvement, while at the same time beams and EFTT were also reduced. With three collimators, the PTV coverage was improved by increasing the PTV $\mathrm{D}_{\min }$ and $\mathrm{D}_{98 \%}$ and decreasing the PTV Dmax, $\mathrm{D}_{2 \%}$, and HI. These findings agreed with the data of Fuller et al., who demonstrated that multiple collimator sizes improved the plan quality (18). However, a similar plan quality can be obtained by using three collimator sizes, instead of all 12, with a smaller incremental increase (19). Our results indicated that in planning for separate PTVs, two Iris collimators can provide similar quality to three collimators. It is critical to consider not only the conformity and homogeneity of the PTV but also the protection of the OARs, because increasing the number of Iris collimators usually results in limited improvement of dose coverage for PTV and plan quality (HI) with longer treatment times but worsens the dosimetry of normal brain. Therefore, the collimator number should be adjusted to allow a good balance between the treatment target and the OARs.

Since we knew that different optimization strategies and object function parameters would affect the plans across many results, all cases in this study were normalized for consistency parameters. However, according to our experience, when the dose volume lower limit (DVL) is used to optimize the PTV, the treatment time will be significantly increased, but this has the same effect for either planning approach. However, we cannot guarantee that the two methods increase the time in the same proportion, and this is one of the limitations of this study. In addition, the lens and brainstem were avoided at beam intersections, which meant that the dose of these OARs was not affected by the planning approaches. This also indirectly shows that the real differences between the two approaches are the difference in the selection of collimators and the difference in the penetration path of the beam on the target.

Although planning for separate lesions clearly showed better performance for delivery efficiency, normal tissue sparing, and plan quality, the total number of delineable organs (including targets and OARs) in the TPS was limited. Therefore, when there are more than six lesions, some workarounds that do not impact the quality of the plan can be applied, such as using both eyes, rather than each eye separately, so that there can be one more target. On the other hand, planning for separate PTVs is not suitable for cases where there are more OARs and lesions that need to be clearly defined.

There were several more limitations of this study. The EFTTs were overestimated due to the lack of time reduction, and other OARs, including the lens, brainstem, and nerves, were not evaluated because the dose was far below the limitation with
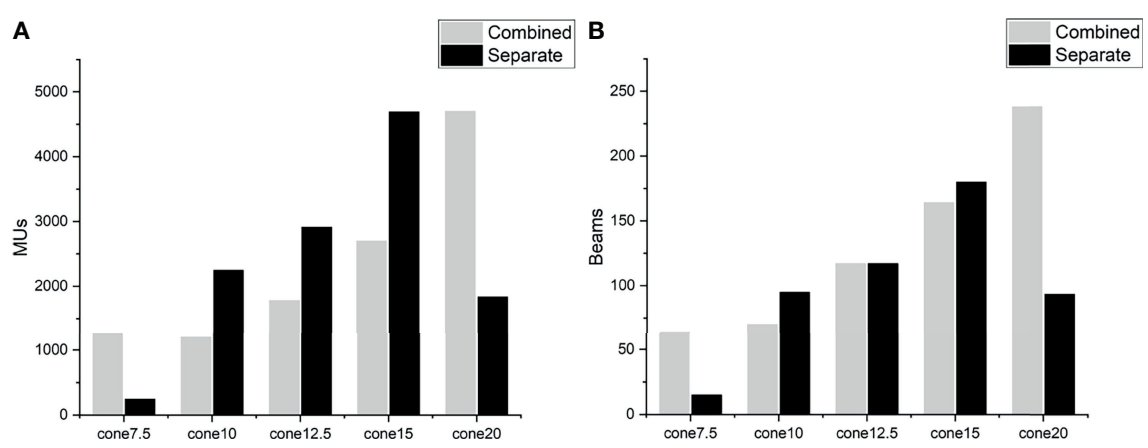

FIGURE 2 | Comparison of MUs (A) and beams (B) in different optimization approaches. 
two approaches by the avoidance of setting in the model of beam intersection.

\section{CONCLUSION}

Both approaches can achieve high-quality treatment plans for SRS/HFSRT in MBM. The Separate approach improved treatment efficiency and dose for normal tissues. The increased number of collimators with the Separate approach decreased treatment efficiency and increased brain dose but improved PTV coverage and plan quality. The selection of the number of collimators must balance PTV coverage and normal tissue sparing.

\section{REFERENCES}

1. Tsao MN, Rades D, Wirth A, Lo SS, Danielson BL, Gaspar LE, et al. Radiotherapeutic and Surgical Management for Newly Diagnosed Brain Metastasis(Es): An American Society for Radiation Oncology EvidenceBased Guideline. Pract Radiat Oncol (2012) 2:210-25. doi: 10.1016/ j.pro.2011.12.004

2. Mengue L, Bertaut A, Ngo ML, Doré M, Ayadi M, Clément-Colmou K, et al. Brain Metastases Treated With Hypofractionated Stereotactic Radiotherapy: 8 Years Experience After Cyberknife Installation. Radiat Oncol (2020) 15:82. doi: 10.1186/s13014-020-01517-3

3. Brown PD, Jaeckle K, Ballman KV, Farace E, Cerhan JH, Anderson SK, et al. Effect of Radiosurgery Alone vs Radiosurgery With Whole Brain Radiation Therapy on Cognitive Function in Patients With 1 to 3 Brain Metastases: A Randomized Clinical Trial. JAMA (2016) 316:401-9. doi: 10.1001/ jama.2016.9839

4. Minniti G, Scaringi C, Paolini S, Lanzetta G, Romano A, Cicone F, et al. Single-Fraction Versus Multifraction $(3 \times 9$ Gy) Stereotactic Radiosurgery for Large $(>2 \mathrm{Cm})$ Brain Metastases: A Comparative Analysis of Local Control and Risk of Radiation-Induced Brain Necrosis. Int J Radiat Oncol Biol Phys (2016) 95:1142-8. doi: 10.1016/j.ijrobp.2016.03.013

5. Yamamoto M, Serizawa T, Shuto T, Akabane A, Higuchi Y, Kawagishi J, et al. Stereotactic Radiosurgery for Patients With Multiple Brain Metastases (JLGK0901): A Multi-Institutional Prospective Observational Study. Lancet Oncol (2014) 15:387-95. doi: 10.1016/S1470-2045(14)70061-0

6. Zhang S, Yang R, Shi C, Li J, Zhuang H, Tian S, et al. Noncoplanar VMAT for Brain Metastases: A Plan Quality and Delivery Efficiency Comparison With Coplanar VMAT, IMRT, and CyberKnife. Technol Cancer Res T (2019) 18:1180676262. doi: 10.1177/1533033819871621

7. El Shafie RA, Tonndorf-Martini E, Schmitt D, Celik A, Weber D, Lang K, et al. Single-Isocenter Volumetric Modulated Arc Therapy vs. CyberKnife M6 for the Stereotactic Radiosurgery of Multiple Brain Metastases. Front Oncol (2020) 10:568. doi: 10.3389/fonc.2020.00568

8. de la Peña C, Guajardo JH, Gonzalez MF, González C, Cruz B. CyberKnife Stereotactic Radiosurgery in Brain Metastases: A Report From Latin America With Literature Review. Rep Pract Oncol Radiother (2018) 23:161-7. doi: 10.1016/j.rpor.2018.02.005

9. Cao Y, Zhu X, Ju X, Liu Y, Yu C, Sun Y, et al. Optimization of Dose Distributions of Target Volumes and Organs at Risk During Stereotactic Body Radiation Therapy for Pancreatic Cancer With Dose-Limiting Auto-Shells. Radiat Oncol (2018) 13:1-6. doi: 10.1186/s13014-018-0956-7

10. Xiao F, Chang Y, Zhang S, Yang Z. Integrating CVH and LVH Metrics Into an Optimization Strategy for the Selection of Iris Collimator for Cyberknife Xsight Lung Tracking Treatment. J Appl Clin Med Phys (2021) 22:210-7. doi: $10.1002 / \mathrm{acm} 2.13136$

11. Li T, Ozhasoglu C, Burton S, Flickinger J, Heron DE, Huq MS. A Method to Improve Dose Gradient for Robotic Radiosurgery. I Appl Clin Med Phys (2015) 16:333-9. doi: 10.1120/jacmp.v16i6.5748

\section{DATA AVAILABILITY STATEMENT}

The original contributions presented in the study are included in the article/supplementary material. Further inquiries can be directed to the corresponding author.

\section{AUTHOR CONTRIBUTIONS}

TJ designed the study. YS, XZ, YW, and GL collected the patients' clinical data and delineated the target volume and reviewed the patients' treatment plans. TJ analyzed the data and wrote the paper. All authors contributed to the article and approved the submitted version.

12. Jang SY, Lalonde R, Ozhasoglu C, Burton S, Heron D, Huq MS. Dosimetric Comparison Between Cone/Iris-Based and InCise MLC-Based CyberKnife Plans for Single and Multiple Brain Metastases. J Appl Clin Med Phys (2016) 17:184-99. doi: 10.1120/jacmp.v17i5.6260

13. Nakamura JL, Verhey LJ, Smith V, Petti PL, Lamborn KR, Larson DA, et al. Dose Conformity of Gamma Knife Radiosurgery and Risk Factors for Complications. Int J Radiat Oncol Biol Phys (2001) 51:1313-9. doi: 10.1016/ s0360-3016(01)01757-6

14. Schlaefer A, Schweikard A. Stepwise Multi-Criteria Optimization for Robotic Radiosurgery. Med Phys (2008) 35:2094-103. doi: 10.1118/1.2900716

15. Chang Y, Liu HY, Liang ZW, Nie X, Yang J, Liu G, et al. Dosimetric Effect of Intrafraction Tumor Motion in Lung Stereotactic Body Radiotherapy Using CyberKnife Static Tracking System. Technol Cancer Res Treat (2019) 18:1078127096. doi: 10.1177/1533033819859448

16. Chan MK, Kwong DL, Lee VW, Leung RW, Wong MY, Blanck O. Feasibility Study of Robotic Hypofractionated Lung Radiotherapy by Individualized Internal Target Volume and XSight Spine Tracking: A Preliminary Dosimetric Evaluation. J Cancer Res Ther (2015) 11:150-7. doi: 10.4103/ 0973-1482.138220

17. Varnava M, Sumida I, Mizuno H, Shiomi H, Suzuki O, Yoshioka Y, et al. A New Plan Quality Objective Function for Determining Optimal Collimator Combinations in Prostate Cancer Treatment With Stereotactic Body Radiation Therapy Using CyberKnife. PLoS One (2018) 13:e208086. doi: 10.1371/journal.pone.0208086

18. Fuller DB, Lee C, Mardirossian G. Comparison of Virtual HDR Prostate Treatment Plans Created With Fixed and Variable Aperture Collimators. Int J Radiat Oncol Biol Phys (2009) 3:s685. doi: 10.1016/j.ijrobp.2009.07.1563

19. Echner GG, Kilby W, Lee M, Earnst E, Sayeh S, Schlaefer A, et al. The Design, Physical Properties and Clinical Utility of an Iris Collimator for Robotic Radiosurgery. Phys Med Biol (2009) 54:5359-80. doi: 10.1088/0031-9155/54/ $18 / 001$

Conflict of Interest: The authors declare that the research was conducted in the absence of any commercial or financial relationships that could be construed as a potential conflict of interest.

Publisher's Note: All claims expressed in this article are solely those of the authors and do not necessarily represent those of their affiliated organizations, or those of the publisher, the editors and the reviewers. Any product that may be evaluated in this article, or claim that may be made by its manufacturer, is not guaranteed or endorsed by the publisher.

Copyright (c) 2022 Ji, Song, Zhao, Wang and Li. This is an open-access article distributed under the terms of the Creative Commons Attribution License (CC BY). The use, distribution or reproduction in other forums is permitted, provided the original author(s) and the copyright owner(s) are credited and that the original publication in this journal is cited, in accordance with accepted academic practice. No use, distribution or reproduction is permitted which does not comply with these terms. 